\title{
V. Verzeichnis der Mitglieder der Vereinigung der Deutschen Staatsrechtslehrer.
}

Gegründet am 13. Oktober 1922.

\section{Vorstand.}

1. Sartorius, Dr. Carl, ord. Professor der Rechte, Tübingen, Lustenau.

2. Kelsen, Dr. Hans, ord. Professor der Rechte, Köln, Mehlemerstr. 26.

3. Koellreutter, Dr. Otto, ord. Professor der Rechte, Oberverwaltungsgerichtsrat, Jena, Schaefferstr. 2.

\section{Mitglieder.}

1. Adamowich, Dr. Ludwig, ord. Professor der Rechte, Graz, Halbärthgasse 6 II.

2. Adler, Dr. Franz, Privatdozent der Rechte an der Deutschen Universität in Prag, Prag XI, Sudomêr̂ská 1413.

3. Anschütz, Dr. Gerhard, ord. Professor der Rechte, Geheimer Justizrat, Heidelberg, Ziegelhäuser Landstr. 35.

4. Apelt, Dr. W., Geheimer Regierungsrat, ord. Professor der Rechte, Leipzig, Lortzingstr. 17.

5. Bilfinger, Dr. Carl, ord. Professor der Rechte, Halle a. d. S., Paulusstr. 4.

6. Bornhak, Dr. Conrad, a. o. Professor der Rechte, Geheimer Justizrat, Berlin SW 61, Blücherplatz 2.

7. Bruns, Dr. Viktor, ord. Professor der Rechte, Berlin-Zehlendorf-West, Sven-Hedin-Str. 19.

8. Bühler, Dr. Ottmar, ord. Professor der Rechte, Münster i. W., Dechaneistr. 19.

9. Calker, van, Dr. Wilhelm, ord. Professor der Rechte, Geheimer Justizrat, Freiburg i. Br., Wölflinstr. 13.

10. Dochow, Dr. Franz, a. o. Professor, Heidelberg, Leopoldstraße 37.

11. Dohna, Graf zu, ord. Professor der Rechte, Bonn, Meckenheimer Allee 32.

12. Ebers, Dr. Godehard Josef, ord. Professor der Rechte, Köln-Marienburg, Ulmenallee 124. 
13. Finger, Dr. A., ord. Professor der Rechte, Geheimer Justizrat, Halle a.d. S., Reichardtstr. 2.

14. Fleiner, Dr. Fritz, ord. Professor der Rechte, Zürich, Mythenkai 4.

15. Fleischmann, Dr. Max, ord. Professor der Rechte, Halle a. d. S., Kaiserplatz 14.

16. Forsthoff, Dr. Emst, Privatdozent an der Universität, Freiburg i. B., Thurnseestr. 67.

17. Frisch, Dr. Hans v., früher ord. Professor an der Uंniversität Czernowitz, jetzt Privatdozent an der Universität, Wien VIII, Josefstädter Str. 17.

18. Genzmer, Dr. jur., Dr. phil. h. c. Felix, ord. Professor der Rechte, Ministerialrat a. D., Marburg a.d. L., Wilhelmstraße 52.

19. Gerber, Dr. Hans, o. Professor der Rechte, Tübingen, Brunsstr. 30.

20. Giese, Dr. Friedrich, ord. Professor der Rechte, Frankfurt a. M.-Süd, Waidmannstr. 20.

21. Gmelin, Dr. Hans, ord. Professor der Rechte, GieBen, Nahrungsberg 51.

22. Glum, Dr. H. Friedrich, Professor an der Universität Berlin, Berlin-Dahlem, Ihnestr. 14.

23. Heckel, Dr., ord. Professor der Rechte, Bonn, Buschstr. 58.

24. Helfritz, Dr. jur. et phil., Hans, ord. Professor der Rechte, Geheimer Regierungsrat, Breslau 18, Kleinburgstr. 15 a.

25. Heller, Dr. Hermann, a. o. Professor an der Universität Berlin, Berlin-Schlachtensee, Adalbertstr. 41.

26. Henrich, Walter, Dr. phil. et rer. pol., a. o. Professor für Rechtswissenschaft an der Deutschen Technischen Hochschule in Brünn, Brünn, Jadokstr. 10.

27. Hensel, Dr. Albert, ord. Professor der Rechte, Königsberg i. Pr., Oberteichufer 10 a.

28. Herrfahrdt, Dr. Heinrich, Privatdozent an der Universität, Greifswald, Karlplatz 18.

29. Hettlage, Di. Karl Maria, Privatdozent, Köln-Klettenberg, Brubergstr. 2.

30. Heyer, Dr. F., ord. Professor der Rechte, Bonn, Humboldtstraße 35.

31. Heyland, Dr., a. o. Professor an der Universität Gießen, Rechtsanwalt, Adresse: Frankfurt a. Main-Süd, Gartenstraße 36.

32. Hippel, Dr. Ernst v., ord. Professor der Rechte, Königsberg, Kastanienallee 41.

33. Huber, Dr. Ernst Rudolf, Privatdozent an der Universität, Bonn, Godesberger Str. 6 II. 
34. Hübner, Dr. Rudolf, ord. Professor der Rechte, Geheimer Justizrat, Jena, Beethovenstr. 6.

35. Hugelmann, Dr. Karl, a. o. Professor an der Universität Wien, Klosterneuburg, Lessingstr. 5.

36. Isay, Dr. Emst, Oberverwaltungsgerichtsrat in Berlin, Privatdozent an der Universität Münster i. W., BerlinGrunewald, Egerstr. 12.

37. Jacobi, Dr. Erwin, ord. Professor der Rechte, Leipzig C 1, Straße des 18. Oktober 17.

38. JahrreiB, Dr. Hermann, a. o. Professor an der Universität, Leipzig N 22, Landsberger Str. 1 (jetzt ord. Professor an der Universität Greifswald).

39. Jellinek, Dr. Walter, ord. Professor der Rechte, Heidelberg, Moltkestr. 10.

40. Jerusalem, Dr. Franz W., ord. Professor der Rechte, Jena, Beethovenstr. 15.

41. Kah1, D. Dr. Wilhelm, ord. Professor der Rechte, Geheimer Justizrat, M. d. R., Berlin-Wilmersdorf, Kaiserallee 23.

42. Kaufmann, Dr. Erich, ord. Professor der Rechte in Bonn, Berlin-Nikolassee, Sudetenstr. 54 a.

43. Kelsen, Dr. Hans, ord. Professor der Rechte, Köln, Mehlemerstr. 26.

44. Koellreutter, Dr. Otto, ord. Professor der Rechte, Oberverwaltungsgerichtsrat, Jena, Schaefferstr. 2.

45. Köhler, Dr. Ludwig v., ord. Professor der Rechte, Staatsminister a. D., Tübingen, Hirschauer Str. 6.

46. Köttgen, Dr. Arnold, ord. Professor der Rechte, Greifswald, Steinstr. 44.

47. Kraus, Dr. Herbert, ord. Professor der Rechte, Göttingen, Merkelstr. 11.

48. Kulisch, Dr. Max, ord. Professor der Rechte, Innsbruck, Adolf-Pichler-Str. 8.

49. Laforet, Dr. W., ord. Professor der Rechte, Würzburg, Theaterstr. 2 II.

50. Langer, Dr. Gottfried, a. o. Professor an der Universität Leipzig, o. Professor an der privaten deutschen Hochschule (Herderinstitut) in Riga, Regierungsrat, Leipzig, Sophienplatz 6 III.

51. Lassar, Dr. Gerhard, a. o. Professor der Rechte, Hamburg 37, Werderstr. 19.

52. Laun, Dr. Rudolf, ord. Professor der Rechte, Hamburg 37, Isestr. $123 \mathrm{I}$.

63. Layer, Dr. Max, ord. Professor der Rechte, Mitglied des Verfassungsgerichtshofs, Wien I, Schellinggasse 5.

54. Leibholz, Dr. Gerhard, ord. Professor der Rechte, Göttingen, Dahlmannstr. 13. 
55. Liermann, Dr. Hans, ord. Professor der Rechte, Erlangen, Loewenichstr. 24.

56. Loewenstein, Dr. Karl, Privatdozent an der Universität München, München, Liebigstr. 3.

57. Marschall von Bieberstein, Freiherr, Dr. F., ord. Professor der Rechte, Freiburg i. Br., Dreisamstr. 11.

58. Menzel, Dr. Adolf, Honorarprofessor der Rechte, Vizepräsident des österreichischen Verfassungsgerichtshofes, Wien 19, Windhabergasse $2 \mathrm{~A}$.

59. Merk, Dr. Ludwig, Privatdozent an der Universität, Tübingen, Kaiserstr. 22.

60. Merkl, Dr. Adolf, a.o. Professor der Rechts- und Staatswissenschaften, Wien XIX, Grinzinger Allee $43 \mathrm{a}$.

61. Mirbt, Dr. H., a. o. Professor an der Universität, Göttingen, Düsterer Eichenweg 22.

62. Nawiasky, Dr. Hans, ord. Professor der Rechte, München, Kaulbachstr. 11.

63. Neuwiem, Dr. E., ord. Professor der Rechte, Münster i. W., Studtstr. 17.

64. Oeschey, Dr. Rudolf, a. o. Professor an der Universität, Leipzig C 1, Grassistr. 44 III. Ferienanschrift: München, Schellingstr. 1.

65. Perels, Dr. Kurt, ord. Professor der Rechte, Mitglied des Oberverwaltungsgerichts, Hamburg, Gustav-Freytag-Str. 7.

66. Peters, Dr. Hans, a. o. Professor, Berlin W 15, Ludwigkirchplatz 11.

67. Redlich, Dr. Josef, früher ord. Universitätsprofessor, Minister a. D., Wien XIX, Armbrustergasse 16.

68. Rich ter, Dr. Lutz, a. o. Professor an der Universität, Leipzig W 31, Rochlitzstr. 1.

69. Ritterbusch, Dr. Paul, Privatdozent, Leipzig S 3, Südstraße 36 .

70. Rothenbücher, Dr. Karl, ord. Professor der Rechte, München, Kaiserplatz 12.

71. Ruck, Dr. Erwin, ord. Professor der Rechte, Geheimer Justizrat, Basel-Bottmingen, Neuenruck.

72. Sander, Dr. Fritz, o. ö. Professor des öffentlichen Rechtes an der Deutschen Universität Prag, Prag VII Vinarska 15.

73. Sartorius, Dr. Carl, ord. Professor der Rechte, Tübingen, Lustenau.

74. Scheuner, Dr. Ulrich, Privatdozent, Berlin-Steglitz, Johanna-Stegenstr. 17.

75. Schindler, Dr. Dietrich, a. o. Professor an der Universität Zürich, Zürich - Zollikon. 
76. Schmidt, Dr. Richard, ord. Professor der Rechte, Geheimer Hofrat, Leipzig, Plagwitzer Str. 49 I.

77. Schmitt, Dr. Carl, ord. Professor der Rechte an der Handelshochschule Berlin, Berlin NW. Flotowstr. 5.

78. Schoen, D. Dr. Paul, ord. Professor der Rechte, Geheimer Justizrat, Göttingen, Merkelstr. 5.

79. Schoenborn, Dr. W., ord. Professor der Rechte, Kiel, Bartelsallee 6.

80. Schranil, Dr. Rudolf, ord. Professor an der Universität Prag, Prag VII, 1303.

81. Schüle, Dr. Adolf, Privatdozent, Berlin-Lichterfelde-West, Hortensienplatz 3 II.

82. Smend, Dr. Rudolf, ord. Professor der Rechte, BerlinNikolassee, Teutonenstr. 1.

83. Stier-Somlo, Dr. Fritz, ord. Professor des öffentlichen Rechts und der Politik, Köln-Marienburg, Marienburger Straße 31.

84. Strupp, Dr. Karl, a. o. Professor an der Universität, Frankfurt a. M., Kettenhofweg 139.

85. Tatarin-Tarnheyden, Dr. Edgar, ord. Professor der Rechte, Rostock i. M., Moltkestr. 18.

86. Thoma, Dr. Richard, ord. Professor der Rechte, Geheimer Hofrat, Bonn, Koblenzer Str. 121.

87. Triepel, Dr. Heinrich, ord. Professor der Rechte, Geheimer Justizrat, Berlin-Charlottenburg, Neue Kantstr. 22.

88. Vervier, Heinrich, Dr. jur. und rer. pol., Regierungsrat I. Kl., Privatdozent an der Universität Würzburg, Theresienstr. 13.

89. Waldecker, Dr. Ludwig, ord. Professor der Rechte, Breslau 18, Scharnhorststr. 16.

90. Walz, Dr., Oberbürgermeister, ord. Honorarprofessor an der Universität Heidelberg.

91. Walz, Dr. Gustav Adolf, Privatdozent an der Universität, Marburg, Universitätsstr. $31 \mathrm{I}$.

92. Wenzel, Dr. Max, ord. Professor der Rechte, Erlangen, Hindenburgstr. 14.

93. Wittmayer, Dr. Leo, Tit. a. o. Professor, Ministerialrat, Wien III, Salesianergasse $1 \mathrm{~b}$.

94. Wolff, Dr. Hans, Reg.-Assessor, Privatdozent, Frankfurt a. M.-Höchst, Gerlachstr. 20.

95. Wolgast, Dr. Ernst, ord. Professor der Rechte, Rostock, Alexandrinenstr. 9 a.

96. Wurmbrand, Dr. Norbert, a. o. Professor, Graz, Glacisstraße 57 . 